\title{
Computed tomography angiography for guiding and follow-up of magnesium-bioresorbable scaffold implantation
}

\author{
Maksymilian P. Opolski ${ }^{1} \cdot$ Cezary Kepka ${ }^{2} \cdot$ Wojtek Wojakowski ${ }^{3} \cdot$ Adam Witkowski $^{1}$
}

Received: 24 March 2018 / Accepted: 21 August 2018 / Published online: 4 September 2018

(c) The Author(s) 2018

Sirs:

Numerous studies in the drug-eluting stent era have consistently identified residual stenosis and stent underexpansion as independent predictors of stent restenosis and thrombosis $[1,2]$. In this regard, refined techniques for stent implantation with complete coronary lesion coverage and appropriate stent sizing are indispensable to decrease the risk of stent failure and improve outcomes [3, 4]. Although stand-alone coronary angiography often underestimates lesion length and vessel size compared with intravascular ultrasound and coronary computed tomography angiography (CTA), it is still widely used for guidance of percutaneous coronary intervention (PCI) [5]. Recently, the concept of CTA-guided PCI has emerged as a valuable alternative to coronary angiography to provide reliable measurements of the vessel size and lesion length as well as the visualization of the morphological features of coronary plaque [6-8].

A 68-year-old male with atypical angina and a prior history of diabetes mellitus and hypertension was referred for noninvasive coronary imaging. Coronary computed tomography angiography (coronary CTA) using a thirdgeneration dual-source scanner (SOMATOM Definition Force, Siemens Healthcare) showed severe stenosis with low-attenuation plaque, positive remodeling and spotty calcification in the proximal-to-mid portion of the left anterior

Electronic supplementary material The online version of this article (https://doi.org/10.1007/s00392-018-1362-8) contains supplementary material, which is available to authorized users.

Maksymilian P. Opolski

opolski.mp@gmail.com

1 Department of Interventional Cardiology and Angiology, Institute of Cardiology, Warsaw, Poland

2 Department of Coronary and Structural Heart Diseases, Institute of Cardiology, Warsaw, Poland

3 3rd Department of Cardiology, Upper Silesian Medical Centre, Medical University of Silesia, Katowice, Poland descending artery (LAD) (Fig. 1a, Online Videos 1 and 2). The interventional treatment strategy (including balloons' and scaffold's size) was established according to the measurements of the reference lumen diameters and lesion length on coronary CTA (Fig. 1b). Specifically, the determinant of the nominal scaffold diameter was based on the mean distal reference lumen diameter. Following coronary angiography (Fig. 1c, Online Video 3), predilation with noncompliant high-pressure balloon (Pantera LEO $3.0 \times 12 \mathrm{~mm}$ at $18 \mathrm{~atm}$ ) was performed and magnesium-bioresorbable scaffold (Magmaris $3.5 \times 20 \mathrm{~mm}$ at $14 \mathrm{~atm}$ ) was implanted in proximal-to-mid LAD (Fig. 1d, e). Finally, the proximal optimization technique using non-compliant balloon (NC Quantum $3.5 \times 8 \mathrm{~mm}$ at $20 \mathrm{~atm}$ ) was applied, obtaining optimal angiographic result, along with complete apposition and good expansion of the scaffold in optical coherence tomography (Fig. 1f, g, Online Videos 4 and 5). After 7 months, coronary CTA showed mild atherosclerosis without discernible scaffold struts (Fig. 1h).

Although intravascular ultrasound is the current gold standard to optimize stent implantation and improve clinical outcomes, there is emerging evidence that measurements of the stent diameter and length performed with coronary CTA and intravascular ultrasound are well correlated [9]. Recently, the concept of CTA-guided PCI has been tested in two randomized trials, wherein CTA-assisted stent sizing resulted in significantly longer stent length and numerically larger stent diameter translating into more complete lesion coverage and less residual disease as compared to angiographic guidance alone [6, 7]. This was corroborated in our case, wherein CTA-assisted PCI resulted in longer and larger scaffold compared to quantitative coronary angiography (Online Fig. 1), and rendered optimal immediate and follow-up results. Furthermore, we believe to show the first-in-man CTA follow-up image of the magnesium scaffold that was not only fully interpretable but also suggested significant bioresorption of the scaffold by 7 months while preserving its luminal integrity. This highlights the potential 


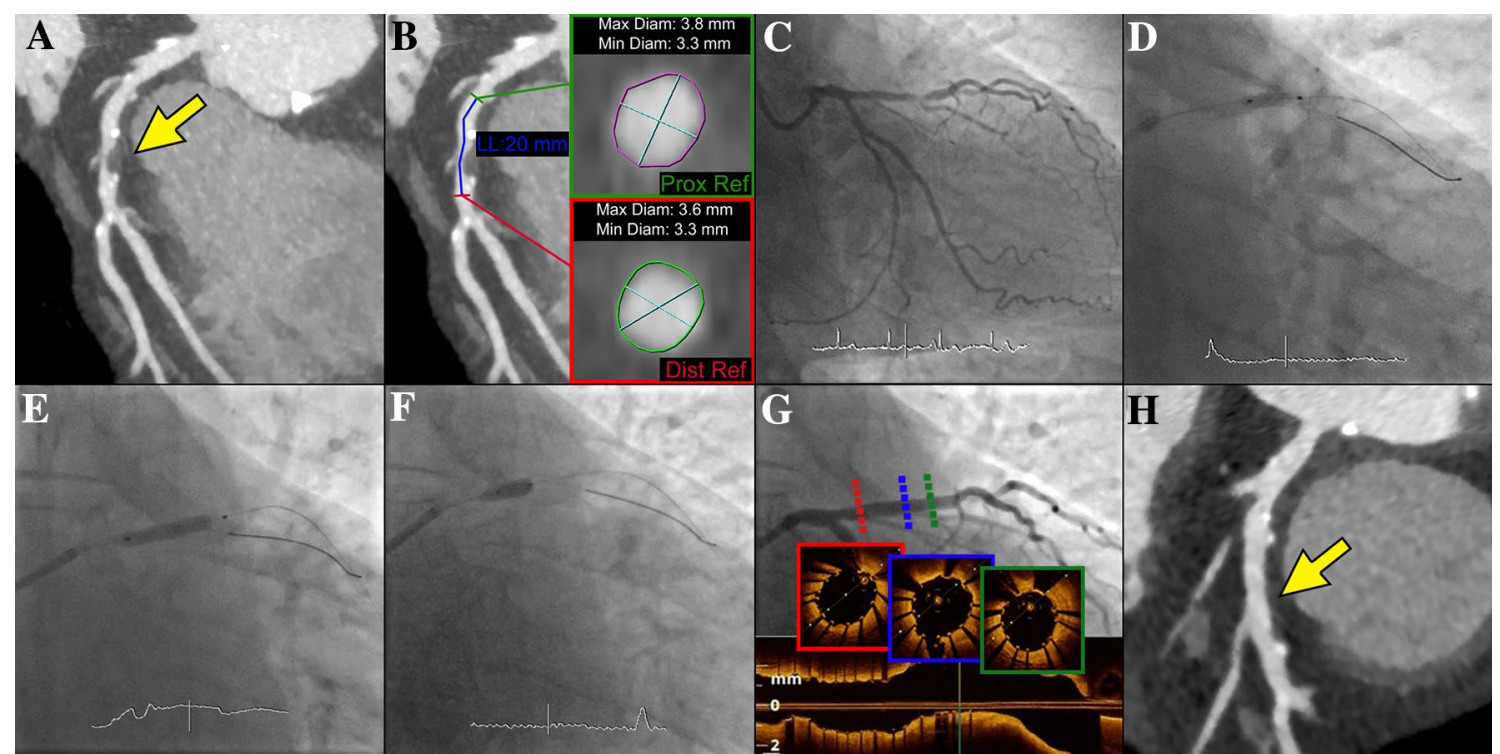

Fig. 1 a Coronary computed tomography angiography (CTA) disclosing severe stenosis (arrow) in the proximal-to-mid left anterior descending artery (LAD) (Online Videos 1 and 2). b CTA-assisted planning of percutaneous coronary intervention (based on computed tomographic data the planned stent length was $20 \mathrm{~mm}$ with proximal and distal mean stent diameters of $3.5 \mathrm{~mm}$ ). c Coronary angiography confirming severe stenosis in the proximal-to-mid LAD (Online Video 3). d Balloon predilation of the LAD using non-compliant high-pressure balloon (Pantera LEO $3.0 \times 12 \mathrm{~mm}$ ). e Magnesium- bioresorbable scaffold (Magmaris $3.5 \times 20 \mathrm{~mm}$ ) implantation in the proximal-to-mid LAD with guidewire protection of the diagonal branch. f Proximal optimization technique using non-compliant balloon (NC Quantum $3.5 \times 8 \mathrm{~mm}$ ) in the proximal portion of the scaffold. $\mathrm{g}$ Final coronary angiography and optical coherence tomography showing optimal angiographic result with complete apposition and good expansion of the scaffold (Online Videos 4 and 5). h Coronary CTA demonstrating mild atherosclerosis without discernible scaffold struts (arrow) in the proximal-to-mid LAD at 7-month follow-up utility of coronary CTA in guiding and follow-up assessment of bioresorbable scaffolds' implantation.

\section{Compliance with ethical standards}

Conflict of interest All authors have neither relevant conflicts of interest nor relationships with industry to disclose.

Open Access This article is distributed under the terms of the Creative Commons Attribution 4.0 International License (http://creativeco mmons.org/licenses/by/4.0/), which permits unrestricted use, distribution, and reproduction in any medium, provided you give appropriate credit to the original author(s) and the source, provide a link to the Creative Commons license, and indicate if changes were made.

\section{References}

1. Sonoda S, Morino Y, Ako J, Terashima M, Hassan AH, Bonneau HN, Leon MB, Moses JW, Yock PG, Honda Y, Kuntz RE, Fitzgerald PJ, SIRIUS Investigators (2004) Impact of final stent dimensions on long-term results following sirolimus-eluting stent implantation: serial intravascular ultrasound analysis from the sirius trial. J Am Coll Cardiol 43:1959-1963. https://doi. org/10.1016/j.jacc.2004.01.044

2. Fujii K, Carlier SG, Mintz GS, Yang YM, Moussa I, Weisz G, Dangas G, Mehran R, Lansky AJ, Kreps EM, Collins M, Stone GW, Moses JW, Leon MB (2005) Stent underexpansion and residual reference segment stenosis are related to stent thrombosis after sirolimus-eluting stent implantation: an intravascular ultrasound study. J Am Coll Cardiol 45:995-998

3. Opolski MP (2018) Cardiac computed tomography for planning revascularization procedures. J Thorac Imaging 33:35-54. https ://doi.org/10.1097/RTI.0000000000000262

4. Blachutzik F, Boeder N, Wiebe J, Mattesini A, Dörr O, Most A, Bauer T, Röther J, Tröbs M, Schlundt C, Achenbach S, Hamm CW, Nef HM (2017) Post-dilatation after implantation of bioresorbable everolimus- and novolimus-eluting scaffolds: an observational optical coherence tomography study of acute mechanical effects. Clin Res Cardiol 106:271-279. https://doi. org/10.1007/s00392-016-1048-z

5. van Velzen JE, de Graaf MA, Ciarka A, de Graaf FR, Schalij MJ, Kroft LJ, de Roos A, Jukema JW, Reiber JH, Schuijf JD, Bax JJ, van der Wall EE (2012) Non-invasive assessment of atherosclerotic coronary lesion length using multidetector computed tomography angiography: comparison to quantitative coronary angiography. Int J Cardiovasc Imaging 28:2065-2071. https://doi.org/10.1007/s10554-012-0015-7

6. Pregowski J, Kepka C, Kruk M, Mintz GS, Kalinczuk L, Ciszewski M, Ciszewski A, Wolny R, Szubielski M, Chmielak Z, Demkow M, Norwa-Otto B, Opolski M, Tyczynski P, Ruzyllo W, Witkowski A (2011) Comparison of usefulness of percutaneous coronary intervention guided by angiography plus computed tomography versus angiography alone using intravascular ultrasound end points. Am J Cardiol 108:1728-1734. https://doi. org/10.1016/j.amjcard.2011.07.043

7. Wolny R, Pregowski J, Kruk M, Kepka C, Mintz GS, Toth GG, Debski A, Ciszewski M, Kukula K, Opolski MP, Chmielak Z, Witkowski A (2017) Computed tomography angiography 
versus angiography for guiding percutaneous coronary interventions in bifurcation lesions - a prospective randomized pilot study. J Cardiovasc Comput Tomogr 11:119-128. https://doi. org/10.1016/j.jcct.2017.01.002

8. Schmermund A, Eckert J, Schmidt M, Magedanz A, Voigtländer $\mathrm{T}$ (2018) Coronary computed tomography angiography: a method coming of age. Clin Res Cardiol 107:40-48. https:// doi.org/10.1007/s00392-018-1320-5
9. Leber AW, Knez A, von Ziegler F, Becker A, Nikolaou K, Paul S, Wintersperger B, Reiser M, Becker CR, Steinbeck G, Boekstegers P (2005) Quantification of obstructive and nonobstructive coronary lesions by 64-slice computed tomography: a comparative study with quantitative coronary angiography and intravascular ultrasound. J Am Coll Cardiol 46:147-154. https ://doi.org/10.1016/j.jacc.2005.03.071 